\title{
Broadside Radar Cross Section of the Perfectly Conducting Cube
}

\author{
ARTHUR D. YAGHJIAN, SENIOR MEMBER, IEEE, AND ROBERT V. MCGAHAN, MEMBER, IEEE
}

\begin{abstract}
The broadside radar cross section (RCS) of the perfectly conducting cube is predicted from arbitrarily low to arbitrarily high frequencies, and compared to measured data taken for cube side lengths ranging from 0.15 to 4 wavelengths. The predicted and measured RCS curves agree to within the estimated experimental limits of accuracy of \pm 1 dB. At low frequencies the magnetic-field integral equation was "augmented" to eliminate its spnrious homogeneous solutions and thus to produce high accuracy beyond the resonance region up through the intermediate frequency range. At high frequencies the conventional diffraction solution was "enhanced" to produce high accuracy down through the intermediate frequency range into the resonance region. Close agreement between these two very different theoretical solutions in the intermediate frequency range confirmed the validity of each solution and permitted calculation of reliable curves for the amplitude and phase of the backscattered far field versus frequency.
\end{abstract}

\section{INTRODUCTION}

$\mathbf{W}$ ITH ITS SIX FACES, 12 edges, and eight corners, the cube presents a challenging problem for the theories and measurement of electromagnetic scattering. The magnetic field in tegral equation (MFIE) solution of Tsai, Dudley, and Wilton [1], for backscattering cross section from a perfectly electrically conducting (PEC) cube under broadside plane-wave incidence, agreed well with the measurements of Ryan [2] up to a cube side length $(s)$ of about 0.5 wavelengths $(\lambda)$. For $s$ beyond about $0.5 \lambda$ the spurious solutions admitted by the MFIE begin to contaminate the numerical solution, and comparisons between theory and experimental measurements become unreliable. To overcome this spurious resonance problem, Yaghjian [3] introduced "aug. mented" integral equations and applied the augmented magneticfield integral equation (AMFIE) to the problem of broadside backscattering from the conducting cube. Because his numerical solution did not exploit the symmetry of the cube, and his matrix inversion subroutine required that the matrix be stored on-line, computer storage capacity limited the accurate solution of the AMFIE to a cube side length of about $1 \lambda$.

Ryan [2], [4] applied the geometrical theory of diffraction (GTD) to determine the high frequency broadside backscattered fields from conducting cubes at harmonically related frequencies which were combined with low frequency experimental data and transformed to obtain time-domain responses. Although no radar cross section (RCS) versus frequency curves were shown in [2] or [4], a similar high frequency diffraction (HFD) solution for broadside RCS is extracted from the enhanced high frequency solution of Section II-G. ${ }^{1}$ Comparison between the HFD curve (Fig. 3) and the AMFIE curve of [3] reveals that there is not very good agreement between the HFD and AMFIE solutions within the region of accuracy ( $s$ less than about $(\lesssim) \lambda)$ of the

Manuscript received January 6, 1984; revised October 26, 1984.

The authors are with the Electromagnetic Sciences Division, Rome Air Development Center, Hanscom AFB, MA 01731.

1 The HFD solution differs from the GTD solution of [2] and [4] because the HFD solution includes doubly diffracted rays (using the term "doubly diffracted" as defined in Section II-E), and because in the limiting procedure used to obtain the broadside RCS from the GTD diffraction coefficients, the fringe current fields from the front face are not entirely recovered.
AMFIE solution. Also, as discussed in Section II-G, inclusion of higher order edge diffracted rays in the HFD solution did not improve the agreement in this region nor enhance the resonance. Moreover, for $s \gtrsim \lambda$ the HFD backscattering solution differs slightly from the physical optics (PO) solution which is plotted in Fig. 4. Thus we have little assurance that the conventional HFD or GTD solution is a significant improvement over the physical optics solution for broadside RCS versus frequency of a cube.

The purpose of the present work is to predict theoretically the broadside backscattering cross section of perfectly conducting cubes with reasonable engineering accuracy $(\approx \pm 1 \mathrm{~dB})$ from arbitrarily low to arbitrarily high frequencies, and to compare the results to measured RCS data covering a frequency range that extends well beyond the resonance region. We take a composite approach starting with an MFIE solution similar to that of [1] for $s$ less than $0.4 \lambda$. For $s$ greater than $0.4 \lambda$ the AMFIE is solved utilizing $x y$ symmetry in order to reduce the number of unknowns and equations by a factor of four, thereby allowing an accurate numerical solution on the available Cyber 750 computer up to a side length of about $1.5 \lambda$.

Beyond about $1.5 \lambda$ an "enhanced high frequency diffraction" (EHFD) solution is used that integrates the currents induced on the leading face and edges of the cube to determine the fields impinging upon the trailing edges. The mutual validity of the EHFD and integral equation solutions is ascertained by their close agreement in the intermediate frequency range $0.4 \lambda \lesssim$ $s \lesssim 1.5 \lambda$. (Also, the EHFD solution reveals clearly the dominant resonance and shows decent agreement with the integral equation solution throughout the resonance region, $s<0.5 \lambda$.)

The composite RCS versus frequency curve (Fig. 8 ) agreed to within about $\pm 1 \mathrm{~dB}$ with measured data taken at $X$-band with aluminum cubes (Fig. 11) ranging in side length from 0.15 to 4 wavelengths. Because of its accuracy over such a broad frequency range, we have been able to use the composite RCS curve as a benchmark solution to evaluate general numerical codes that compute scattering [15].

\section{THE ENHANCED HIGH FREQUENCY DIFFRACTION SOLUTION}

\section{A. Method of Solution}

A time-harmonic $e^{-i \omega t}$ plane wave $E$-polarized in the $y$ direction is incident broadside along the negative $z$-axis of a perfectly electrically conducting cube shown in Fig. 1. The front and back faces of the cube are located at $z=0$ and $z=-s$, the top and bottom faces at $y=+s$ and $y=-s$, and the right and left side faces at $x=+s$ and $x=-s$, respectively. To obtain a high frequency solution, we begin by dividing the currents excited on the surface of the cube into the physical optics current on the front face and the remaining or "fringe currents" emanating from the edges of the cube. Separation of the surface currents into PO currents and fringe currents conforms to the approach of Ufimtsev [5]. Although fringe currents generally become 


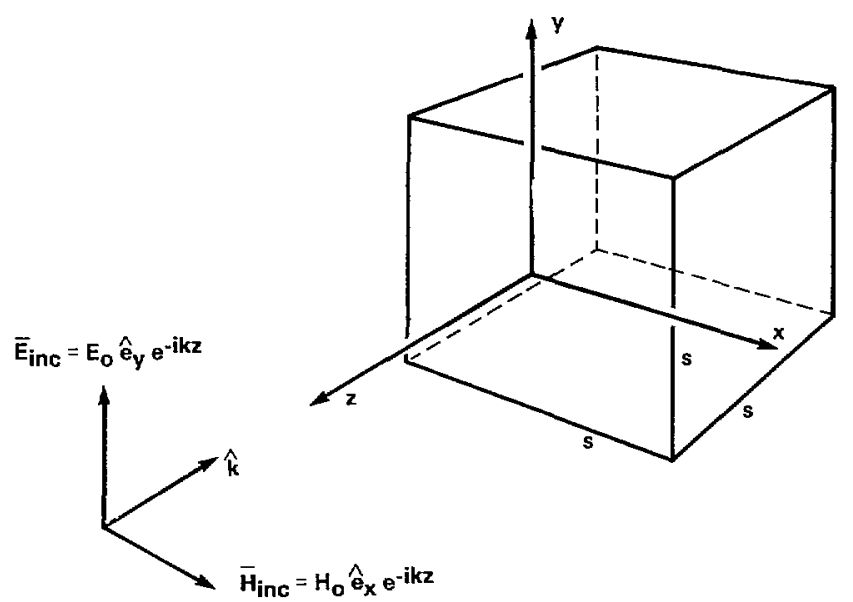

Fig. 1. Perfectly conducting cube with side length $s$ and broadside plane-wave incidence.

negligible within a small fraction of a wavelength from an edge, a notable exception occurs for grazing $H$-wave incidence like that across the top and bottom faces of the cube in Fig. 1, where the fringe current does not approach zero with increasing distance from the leading edge.

The PO surface current is defined as $2 \hat{e}_{z} \times \vec{H}_{\text {inc }}$, i.e., the current on the front face of the cube that would be excited by the incident field if the front face were part of an infinite PEC plane.

For the determination of singly diffracted rays from the front edges, the fringe currents along the leading front four edges of the cube are approximated by the fringe curents (total current minus $\mathrm{PO}$ current) that would emanate from each edge if it were part of the corresponding infinite right-angled wedge illuminated by the incident plane-wave. In the context of GTD, a similar technique for evaluating edge diffracted fields in the vicinity of a caustic region has been called the method of equivalent (edge) currents [6], [7].

To determine the singly diffracted rays from the back edges, the fringe currents emanating from the back four edges of the cube can also be approximated by those of the corresponding infinite right-angled wedges, provided the fields impinging upon these edges can be estimated. The fields impinging upon the trailing edges of the cube consist of the fields scattered by the leading edges in addition to the incident plane-wave field. The usual method of estimating the high frequency fields impinging upon a trailing edge at grazing incidence uses the fields propagated along the grazing face away from the leading edge, under the assumption that the leading edge were part of an infinite wedge. For a right-angled wedge the grazing currents or fields have a simple closed-form expression [8] that approaches the incident plane-wave field for large $s$. Unfortunately, for a cube side length $s$ less than than $1 \lambda$, where meaningful comparison between the high frequency solution and an accurate integral equation solution is possible, the front face of the cube is a poor approximation to the infinite face of the right-angled wedge and the simple right-angled wedge expression for grazing fields is shown in Section II-F to be a poor approximation to the actual surface fields. Moreover, Section II-G shows that this basic high frequency approximation for broadside scattering from the cube remains inadequate regardless of how many interactions (multiply diffracted rays) between the edges of the cube are included in the solution. To overcome this difficulty and enhance the high frequency solution to hold with respectable accuracy even in the resonance region, we use a magnetic field equation to integrate the $\mathbf{P O}$ and fringe currents on the leading face and edges to get a better approximation to the fields impinging upon the trailing edges of the cube.

After the backscattered far fields are obtained in this manner from the PO current and the singly diffracted rays, the high frequency solution is further refined to include the doubly diffracted rays.

Direct influence of any fringe currents existing along the four edges of the cube parallel to the $z$-axis is neglected. Diffraction from the corners of the cube, which is higher order than edge diffraction, is approximated implicitly when the fringe currents are integrated over the finite length of the edges [9].

\section{B. The Physical Optics Field}

We begin the enhanced high frequency diffraction solution by finding the backscattered far fields of the physical optics surface current

$$
\bar{K}^{\mathrm{PO}}=2 \hat{e}_{z} \times \bar{H}_{\text {inc }}=2 H_{o} \hat{e}_{y}
$$

on the front face of the cube, where $H_{0}$ is the amplitude of the incident $H$-field at $z=0$. Insertion of the PO current (1) into the integral for the far-zone magnetic field [10] produces, upon integration, the PO backscattered magnetic far field,

$$
\bar{H}^{\mathrm{PO}}=\frac{-i(k s)^{2}}{2 \pi} H_{o} \frac{e^{i k z}}{k z} \hat{e}_{x},
$$

where $k=2 \pi / \lambda$ is the propagation constant. Any valid far-field solution to the cube should approach the PO result (2) as $k s$ becomes large.

\section{The Singly Diffracted Fields from the Front Edges}

To obtain the singly diffracted far fields of the fringe currents of the four front edges of the cube, we use the Ufimtsev diffraction coefficients [5] $f$ and $g$ (for a right-angled wedge) multiplied by the conversion factor

$$
s \sqrt{\frac{k}{2 \pi z}} e^{-i \pi / 4},
$$

which accounts for the finite length $s$ of the edges of the cube. (This factor is merely the ratio of the far field of a line source of length $s$ to that of an infinite line source.)

Specifically for the plane-wave incidence in Fig. 1, the $H$ far field produced in the backscatter direction by the fringe currents of the edges at $(x= \pm s / 2, z=0)$ is found from the $E$-wave coefficient $f$ to be

$$
\bar{H}_{1}^{x o}=\frac{-k s}{\pi \sqrt{3}} H_{o} \frac{e^{i k z}}{k z} \hat{e}_{x} .
$$

The subscript one denotes singly diffracted fields and the superscripts $x$ and $o$ denote the $\mathbf{x}$ edges on the front face $z=\mathbf{0}$.

Similarly, the singly diffracted backscattered $H$-field from the fringe currents of the front edges of the cube at $(y= \pm s / 2$, $z=0$ ) is found from the $H$-wave coefficient $g$

$$
\bar{H}_{1}^{y o}=\frac{-k s}{\pi 3 \sqrt{3}} H_{o} \frac{e^{i k z}}{k z} \hat{e}_{x} .
$$

\section{The Singly Diffracted Fields from the Back Edges}

The far fields scattered by the back edges of the cube can also be found simply from the diffraction coefficients, provided the grazing field impinging upon the back edges of the cube is known. Consider first the top and bottom rear edges at $(y=$ $\pm s / 2, z=-s$ ). Initially assume an average magnetic field impinging upon these edges given by $H_{o}^{\prime}$ exp (iks), but postpone 
the evaluation of $H_{o}^{\prime}$ until the later Section II-F. A convenient way to determine the far fields of these back two edges is to note that only the fringe current on the back faces of these edges contributes to the backscattered far field; thus the desired backscattered $H$ far field will be just the negative of the forward scattered field. Since this forward scattered $H$-field is easily found from the $H$-wave diffraction coefficient $g$, the backscattered $H$ far field from the top and bottom back edges is readily determined as

$$
\bar{H}_{1}^{y s}=\frac{-k s}{\pi 3 \sqrt{3}} H_{o}^{\prime} e^{2 i k s} \frac{e^{i k z}}{k z} \hat{e}_{x} .
$$

The $e^{2 i k s}$ phase factor, present in (5) but missing in (4), accounts for the extra propagation path length to and from the back of the cube. The diffracted field (5) can also be found from the more conventional GTD procedure of taking half the value of the GTD $H$-wave diffraction coefficient when the direction of propagation lies along a terminated conducting plane [11]. To the field in (5) could be added the secondary scattered field of the current excited on the front face of the leading edges by the return field diffracted from the back edges. This doubly diffracted field will be determined along with the other doubly diffracted rays, in the next section.

The grazing field traveling toward the left and right rear edges of the cube is greatly diminished by propagation along the perfectly conducting sides parallel to the electric field of the incident plane wave. In other words, for the plane-wave incidence of Fig. 1, the side faces severely shadow the back edges at $(x= \pm s / 2, z=-s)$ to the extent that diffraction from these edges can be neglected. To prove that this is indeed the case even for $k s$ on the order of one, we use $[8,(6.13)]$ to estimate the current impinging upon the rear side edges, then [8, eq. (6.10)] to find the diffracted $H$-field returning from an equivalent grazing plane wave that would produce the impinging current, and finally the $f$-diffraction coefficient to find the diffracted far field from the front side edges subject to the returning grazing $H$-field. In all we find an $H$ far field diffracted from the rear side edges given approximately by

$$
\bar{H}_{1}^{x s}=\frac{k s}{\pi 3 \sqrt{3}} \sqrt{\frac{2}{\pi}} \frac{i e^{i \pi / 4}}{8 k s} H_{o} e^{2 i k s} \frac{e^{i k z}}{k z} \hat{e}_{x} .
$$

Because $\bar{H}_{1}^{x s}$ is less by over an order of magnitude than the far field $\bar{H}_{1}^{y s}$ diffracted from the top and bottom back edges when $k s$ is greater than unity, and because it becomes increasingly smaller with increasing $k s$ than even the doubly diffracted terms (derived in the next section), $\bar{H}_{1}^{x s}$ will not be included in the EHFD solution.

\section{E. The Doubly Diffracted Fields}

The doubly diffracted rays are incident rays that diffract from two edges before returning to the backscatter direction. For the cube under broadside incidence, they consist of three sets of rays: 1) those that travel once between opposite edges of the front face, 2) those that travel once between opposite edges of the back face, and 3) those secondary rays mentioned in Section II-D that are excited at the front edges by the rays returning from the corresponding trailing edges. To obtain the field of each doubly diffracted ray, the applicable diffraction coefficient is used twice--the first time to determine the diffracted field radiating from the first edge toward the second edge, and the the second time to evaluate the backscattered field radiated by the second edge under the illumination by the fields from the first edge. Since the three sets of doubly diffracted fields are evaluated by a straightforward manipulation of the diffraction coefficients, only the results will be given here.

Letting $\bar{H}_{2}^{y o}, \bar{H}_{2}^{y s}$, and $\bar{H}_{2}^{y z}$ designate the $H$-wave magnetic field of the three sets of doubly diffracted rays numbered above as 1), 2), and 3), respectively, we find

$$
\begin{aligned}
& \bar{H}_{2}^{y o}=\Delta \frac{k s}{\pi \sqrt{3}} H_{o} e^{i k s} \frac{e^{i k z}}{k z} \hat{e}_{x} \\
& \bar{H}_{2}^{y s}=\Delta \frac{k s}{\pi \sqrt{3}} H_{o}^{\prime} e^{3 i k s} \frac{e^{i k z}}{k z} \hat{e}_{x} \\
& \bar{H}_{2}^{y z}=-\Delta \frac{k s}{\pi 9 \sqrt{3}} H_{o}^{\prime} e^{2 i k s} \frac{e^{i k z}}{k z} \hat{e}_{x} .
\end{aligned}
$$

The double diffraction factor $\Delta$ in (7a)-(7c) equals $\sqrt{2 /(3 \pi k s)} e^{i \pi / 4}$

Similarly, designate the doubly diffracted $E$-wave magnetic fields by $\bar{H}_{2}^{x o}, \bar{H}_{2}^{x s}, \bar{H}_{2}^{x z}$. The fields $\bar{H}_{2}^{x s}$ and $\bar{H}_{2}^{x z}$ from the doubly diffracted rays can be eliminated immediately because the singly diffracted fields exciting these rays propagate across the faces $x= \pm s / 2$ parallel to the $E$-field and were shown in Section II-D to have negligible magnitude for $k s$ greater than about one. The field $\bar{H}_{2}^{x o}$ of the $E$-wave rays diffracted across the front face of the cube from the edge at $x=\mp s / 2$ to the edge at $x=$ $\pm s / 2$ has the considerably higher value

$$
\bar{H}_{2}^{x o}=\frac{\Delta}{i \pi 3 \sqrt{3}} H_{0} e^{i k s} \frac{e^{i k z}}{k z} \hat{e}_{x} .
$$

However, $\bar{H}_{2}^{x o}$ also will not be included in the EHFD solution because it decays with $k s$ faster than the $H$-wave doubly diffracted terms (7), and computations show that it has negligible effect on the final solution even for $k s$ smaller than one.

\section{F. Evaluation of the Fields Impinging Upon the Top and Bottom Trailing Edges}

The scattered far fields given by (5), (7b), and (7c) require the total magnetic field $H_{o}^{\prime} \exp (i k s)$ illuminating the top and bottom trailing edges of the cube. As a first approximation to $H_{o}^{\prime}$ one might use the value of the total field emanating from the top and bottom leading edges of the cube assuming that each of these leading edges is part of an infinite right-angled wedge. Specifically, the solution to the right-angled wedge [8] reveals that this total grazing field behavies asymptotically (with large distance $k s$ from the edge) as

$$
H_{o}^{\prime} \sim H_{o}\left(1+\frac{\Delta}{3}\right) \rightarrow H_{o} .
$$

For a right-angled wedge, the expression (9) for the grazing field is reasonably accurate even for $k s$ on the order of unity. However, evaluation of (15) below shows that its useful accuracy for the cube is limited to much larger values of $k s$. This is because the approximation (9) to $H_{o}^{\prime}$ becomes accurate for the cube only when the PO surface current on the front face of the cube extends over a large enough area to produce the grazing fields of the infinite front face of the right-angled wedge.

To get an approximation for $H_{o}^{\prime}$ more accurate than (9), we use an integral formulation for the magnetic field in terms of surface current on the cube. In particular, the total magnetic field ( $H_{o}^{\prime} \exp (i k s) \hat{e}_{x}$ ) at a distance $k s$ along the center of the top or bottom surface $(x=0, y= \pm s / 2, z=-s)$ of the cube for the 
broadside plane-wave incidence of Fig. 1 is expressed conveniently by the magnetic field equation as [3]

$$
\begin{gathered}
H_{o}^{\prime} e^{i k s \hat{e}_{x}=2 H_{o} e^{i k s} \hat{e}_{x}}+\frac{\hat{e}_{z}}{2 \pi} \times \frac{\partial}{\partial z} \int_{S_{o}} \bar{K}\left(\bar{r}^{\prime}\right) \psi\left(\bar{r}^{\prime}, \bar{r}_{o}\right) d S^{\prime}, \\
\left(\bar{r}_{o}= \pm \frac{s}{2} \hat{e}_{y}-s \hat{e}_{z}\right),
\end{gathered}
$$

where $\bar{K}$ is the surface current, $\psi\left(\bar{r}^{\prime}, \bar{r}\right)$ is the scalar Green's function exp $\left(i k\left|\bar{r}^{\prime}-\bar{r}\right| /\left|\bar{r}^{\prime}-\bar{r}\right|\right)$, and $S_{o}$ is the surface of the cube excluding the top or bottom face on which $\bar{r}_{o}$ is located. Because $H_{o}^{\prime}$ refers to the magnetic field impinging upon the top or bottom trailing edge of the cube, the current $\bar{K}$ includes only the PO and fringe currents of the front face and leading edges. In other words, $H_{o}^{\prime} \exp (i k s)$ is the total magnetic field that would exist at the surface point $\bar{r}_{o}$ of the cube, if the sides of the cube continued to infinity past the trailing edges.

The major contribution to the integral in (10) is from the PO current on the front face of the cube. Denoting this integral by $\bar{I}^{\mathrm{PO}}$, i.e.,

$$
\bar{I}^{\mathrm{PO}}=\frac{\hat{e}_{z}}{2 \pi} \times \frac{\partial}{\partial z} \int_{-s}^{0} \int_{-s / 2}^{s / 2} \vec{K}^{\mathrm{PO}} \psi\left(\bar{r}^{\prime}, \bar{r}_{o}\right) d x^{\prime} d y_{o}^{\prime} .
$$

$$
\left(y_{o}^{\prime} \equiv y^{\prime}-s / 2\right),
$$

we find upon substitution of $\bar{K}^{\text {PO }}$ from (1) into (11) that

$$
\bar{I}^{\mathrm{PO}}=\hat{e}_{x} H_{o} e^{i k s}\left[-1+\frac{2}{\pi} e^{-i k s} \int_{0}^{\pi / 2} \frac{e^{i k s \alpha_{o}(\phi)}}{\alpha_{o}(\phi)} d \phi\right]
$$

The function $\alpha_{o}(\phi)$ is defined as

$$
\alpha_{o}(\phi) \equiv\left\{\begin{array}{ll}
\sqrt{1+\sec ^{2} \phi / 4}, & 0 \leqslant \phi \leqslant \tan ^{-1} 2 \\
\sqrt{1+\csc ^{2} \phi}, & \tan ^{-1} 2 \leqslant \phi \leqslant \pi / 2
\end{array}\right\} .
$$

The double integration of (11) reduces to the single integration of (12a) after converting the $x^{\prime}, y_{o}^{\prime}$ integration variables to spherical coordinates $\left(R^{\prime} \sin \theta \cos \phi, R^{\prime} \sin \theta \sin \phi\right)$ centered at the observation point $\bar{r}_{o}$ (so that the area element equals $R^{\prime 2} \sin \theta d \theta d \phi$ and $R^{\prime}$ equals $s / \cos \theta$ ), and performing the $\theta$ integration. The remaining $\phi$ integration of (12a) is done numerically in a short subroutine of the computer program for evaluating the EHFD solution.

In addition to the PO current $\bar{K}^{\mathrm{PO}}$, the fringe currents associated with the four leading edges of the cube contribute to the integral in (10) for the field impinging upon the top and bottom trailing edges. However, a rough estimate of the contribution from the fringe currents of the top or bottom front edge to the opposite back edge shows that it is small compared with the integral in (12a), and thus can be neglected in the determination of $H_{o}^{\prime}$.

To obtain the magnetic field impinging upon the top or bottom trailing edge from the fringe current of its respective leading edge, we note that for an infinite right-angled wedge $\alpha_{o} \rightarrow \infty$, the integral in (12a) becomes negligible, and the $H_{o}^{\prime}$ contribution of the PO current reduces to simply $H_{0}$. Thus the $\Delta / 3$ term in the large $k s$ expression (9) for $H_{o}^{\prime}$ must be produced by the fringe current of the respective leading edge. Labeling this contribution to the integral in (10) by $\bar{I}^{y}$, we have

$$
\tilde{I}^{y}=H_{0} \frac{\Delta}{3} e^{i k s} \hat{e}_{x}
$$

The contribution (13) to the field impinging upon a trailing edge from the leading-edge fringe current can also be obtained from the fringe diffraction coefficient $g$ by realizing that the magnetic field radiated by the fringe current toward the trailing edge is minus twice the magnetic field radiated in the opposite direction.

The final contribution to the current integral in (10) is from the fringe currents along the front side edges at $(x= \pm s / 2, z=0)$. At first thought one might argue that these side fringe currents contribute negligibly to the fields on the top and bottom faces because they radiate like cylindrical waves over the side faces. Indeed this is true for large $k s$, but for $s \lesssim \lambda / 2(k s \lesssim \pi)$ the fringe currents on these side edges will also radiate over the top and bottom faces of the cube. We can estimate the contribution of the front side fringe currents to the integral in $(10)$ by judiciously applying the Ufimtsev diffraction coefficient $f$. Because the field of the fringe current of the corresponding right-angled wedge is analytic except in the direction of the face of grazing incidence, the diffraction coefficient $f$ is analytic throughout the same region and will give the field produced by the fringe current inside the $90^{\circ}$ wedge angle as well as outside. Use of this property of $f$ allows the integral in (10) of the fringe currents along the front side edges to be approximated by

$$
\bar{I}^{x} \approx 2 \sqrt{3} f_{0} F_{s} \Delta e^{i k s} H_{o} \hat{e}_{x},
$$

where $f_{o}$, the $E$-wave diffraction coefficient in the direction of $\bar{r}_{o}$, equals approximately $1 / 2$. The spreading factor $F_{s}$ is given approximately by

$$
F_{s} \approx \sqrt{\frac{k s}{2 \pi}}\left(1+\frac{i}{k s}\right) e^{-i \pi / 4}\left(\frac{\sin (k s / 4)}{k s / 4}\right)^{2},
$$

and accounts for the finite length and angular displacement of the radiating edges. The $\sin (k s / 4) /(k s / 4)$ part of the spreading factor occurs twice-once to account for the field point $\bar{r}_{o}$ on the top or bottom face lying at an angle roughly equal to 0.5 rad off boresight of the radiating edges, and a second time to account for the linear phase variation of the radiated field across the trailing edges. (The first portion of the spreading factor, i.e., $\sqrt{k s / 2 \pi}(1+i / k s) e^{-i \pi / 4}$ in $(14 \mathrm{~b})$, is an asymptotic result that holds for $k s \lesssim 2$; however, because $\bar{I}^{x}$ has negligible effect on the total solution for $k s>2$, regardless of whether the exact or asymptotic form of (14b) is used, the simpler asymptotic result in (14b) is retained for all values of $k s$.)

In all, $H_{o}^{\prime}$ is found by adding the individual current contributions (12). (13), and (14) comprising the integral in (10)

$$
\begin{aligned}
H_{o}^{\prime}= & H_{o} F_{o}^{\prime}=H_{o}\left[1+\frac{2}{\pi} e^{-i k s} \int_{0}^{\pi / 2} \frac{e^{i k s \alpha_{o}(\phi)}}{\alpha_{o}(\phi)} d \phi\right. \\
& \left.+F_{s} \Delta \sqrt{3}+\frac{\Delta}{3}\right] .
\end{aligned}
$$

The factors $\Delta, \alpha_{o}(\phi)$, and $F_{s}$ are defined in (7), (12b) and (14b), respectively. Note that for large $k s$, the amplitude $H_{o}^{\prime}$ of the magnetic field impinging upon the top and bottom trailing edges reduces to $H_{O}$, the asymptotic amplitude (9) of the grazing magnetic field propagating over the top of the corresponding right-angled wedge. Using $H_{o}^{\prime}$ instead of $H_{0}$ in our high frequency solution distinguishes the EHFD solution from an ordinary HFD solution or the usual GTD solution [2], [4], [11] and, as the next section shows, extends the useful range of applica- 
tion of the high frequency solution to frequencies in the resonance region.

\section{G. Numercial Results for the High Frequency Solutions}

Now that the physical optics far field has been determined in Section II-B, the singly diffracted far fields in Sections II-C and II-D the doubly diffracted far fields in Section II-E, and the grazing field $H_{o}^{\prime}$ illuminating the top and bottom trailing edges in Section II-F, the EHFD magnetic far field $\left(\bar{H}_{\mathrm{sc}}^{\text {cube }}\right)$ backscattered by the cube under the broadside plane-wave incidence of Fig. 1 can be written

$$
\begin{aligned}
\bar{H}_{\mathrm{sc}}^{\mathrm{cube}}= & \hat{e}_{x} H_{o} \frac{k s}{3 \pi \sqrt{3}}\left[\frac{3 \sqrt{3 k s}}{2 i}+\left(2-F_{o}^{\prime} e^{2 i k s}\right)\right. \\
& \left.+\Delta\left(3 e^{i k s}-\frac{F_{o}^{\prime}}{3} e^{2 i k s}+3 F_{o}^{\prime} e^{3 i k s}\right)\right] \frac{e^{i k z}}{k z} .
\end{aligned}
$$

The physical optics, singly diffracted, and doubly diffracted fields are given, respectively, by the first, second, and third term within the brackets of (16). The "double-diffraction factor" $\Delta$ is defined under (7), and the "enhancement factor" $F_{o}^{\prime}$ is defined by (15).

The radar cross section $\sigma$, which is related to $\bar{H}_{\text {sc }}^{\text {cube }}$ by the simple expression

$$
\sigma=\frac{4 \pi z^{2}\left|\bar{H}_{\mathrm{sc}}^{\text {cube }}\right|^{2}}{H_{o}^{2}},
$$

is computed using the EHFD solution (16) and plotted in Fig. 2 as a function of the perimeter $(4 s / \lambda)$ of the cube. Comparison of Fig. 2 with the method of moments solution for the magnetic field integral equations of Section III (Fig. 7) shows that the two RCS curves agree closely for $1.5<4 s / \lambda<6$, and even reasonably well in the resonance region $(4 s / \lambda \leqslant 1.5)$ where a high frequency diffraction solution is not expected to yield valid results.

Fig. 3 reveals that this unexpected agreement in the resonance region occurs because $H_{o}^{\prime}$ is used instead of $H_{o}$ for the fields impinging upon the top and bottom trailing edges. The curve in Fig. 3 is the RCS computed from the less accurate high frequency approximation of using $H_{o}$ for the grazing field at the trailing edges. The variations of this conventional HFD solution in Fig. 3 are less pronounced than the EHFD solution, the resonance is hardly discernable, and the distinction between the conventional HFD solution and the PO solution shown by the curve in Fig. 4 is considerably less than between the EHFD and PO solutions.

Fig. 5 plots only the PO plus single-diffraction terms of the EHFD solution (16) in order to show the importance of the contribution of the doubly diffracted rays. Expressions for the far fields of the triply diffracted rays were also determined, but are not shown because they had no perceptible effect on either the EHFD curve of Fig. 2 or the conventional solution curve of Fig. 3. This negligible effect of the triply diffracted rays on broadside RCS supports the conclusion that the conventional high frequency solution is limited by the use of $H_{o}$ for the fields grazing upon the trailing edges and not by the neglect of multiply diffracted rays of order higher than two.

\section{THE MAGNETIC-FIELD INTEGRAL EQUATION SOLUTION}

The MFIE has been solved numerically for the broadside RCS of the cube by Tsai, Dudley, and Wilton [1], and more

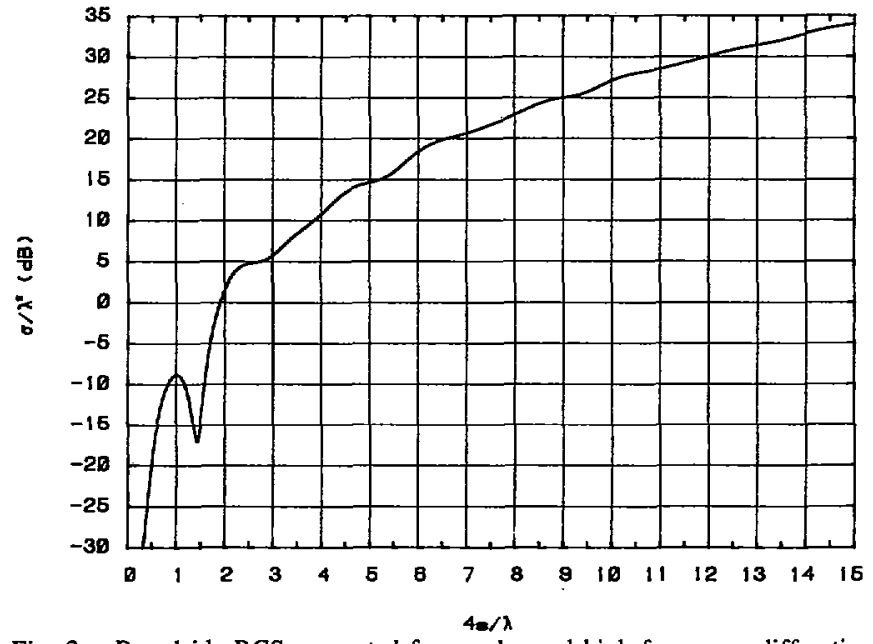

Fig. 2. Broadside RCS computed from enhanced high frequency diffraction (EHFD) solution.

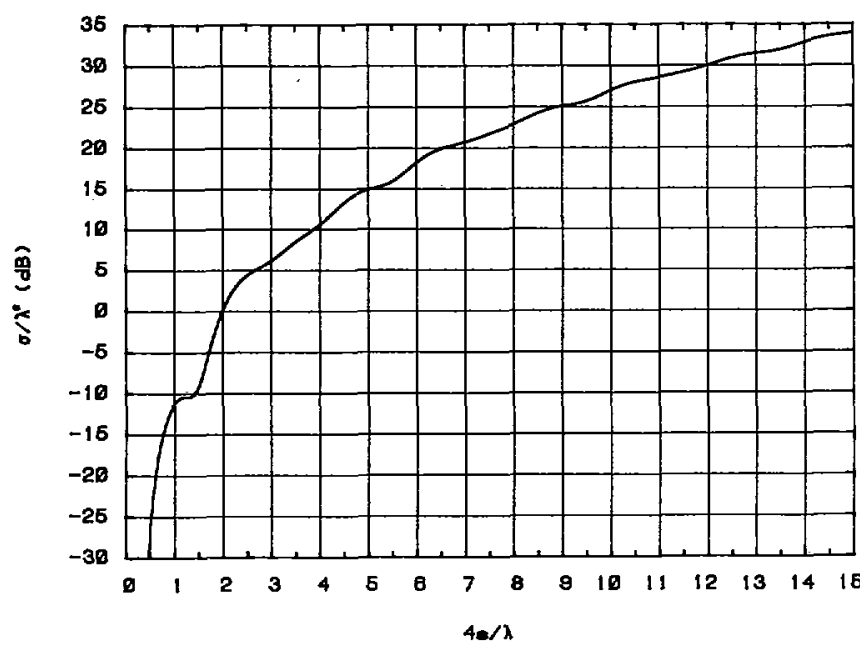

Fig. 3. Broadside RCS computed from conventional high frequency diffraction (HFD) solution.

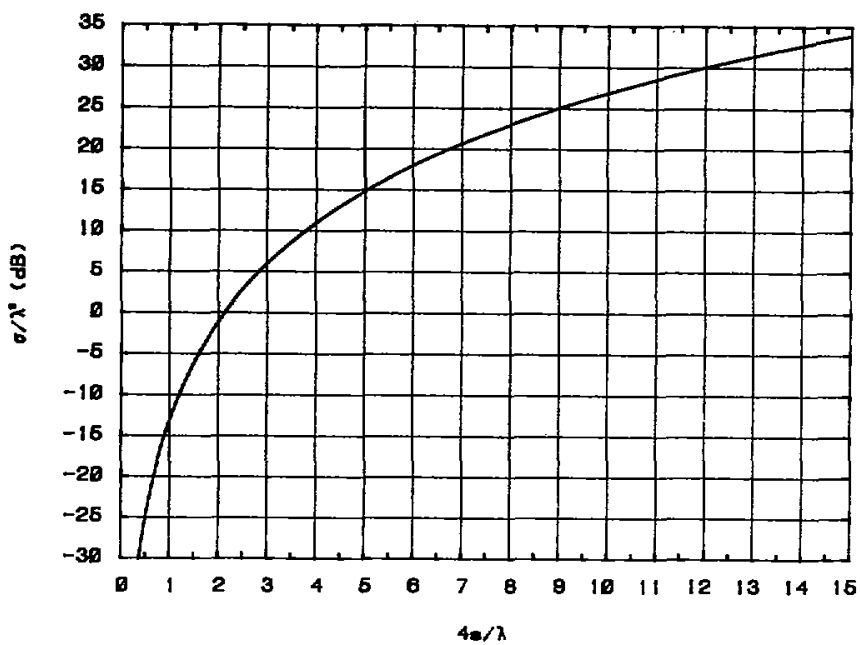

Fig. 4. Broadside RCS computed from physical optics solution. 


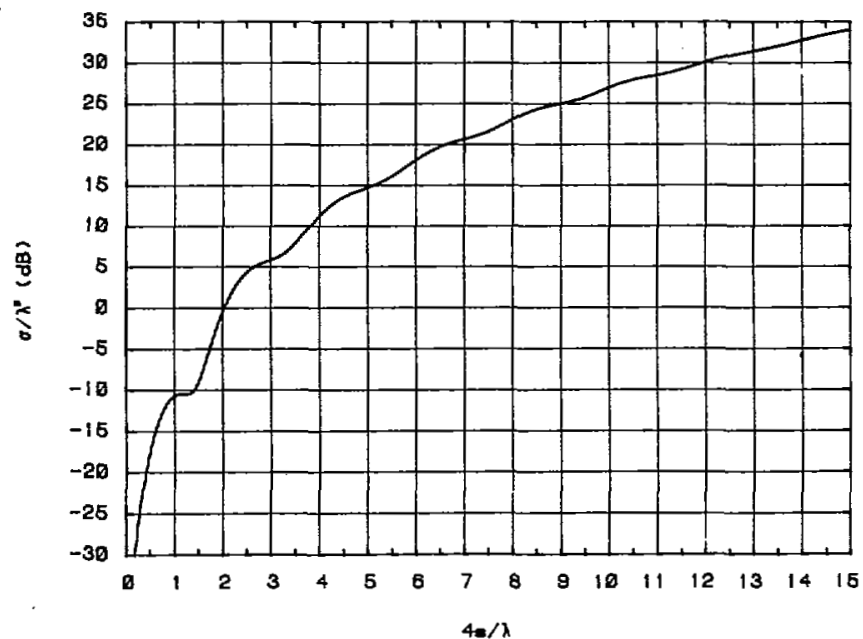

Fig. 5. Broadside RCS computed from EHFD solution without doubly diffracted rays.

recently by Wang and Drane [12], up to a cube side length of about $0.7 \lambda$. Unfortunately, the MFIE (and, incidentally, the electric-field integral equation EFIE) suffers from spurious homogeneous solutions at the resonant frequencies of the corresponding interior cavity [3], [13], [14]. The first interior resonant frequency of the cube occurs at $s / \lambda=0.7$, and subsequent resonance frequencies are spaced at increasingly narrower frequency intervals. Consequently, for $s / \lambda$ greater than about 0.5 , numerical solutions to the MFIE rapidly deteriorate. Fig. 6 reveals the severity of the spurious resonance problem by plotting the numerical MFIE solution for RCS out to a cube side length of about 4 . The numerical scheme that was used is identical to that of [1] with 384 patches over the cube. The jagged peaks and valleys in the RCS curve of Fig. 6 are caused by the spurious solutions of the MFIE at the in terior resonant frequencies.

A number of methods have been devised to eliminate the spurious resonances for the MFIE (and EFIE) solution (see [3] for a survey of the various methods). A particularly simple technique [3] for eliminating the spurious solutions "augments" the original magnetic-field integral equation with the condition that the normal $H$-field be zero at the surface of the perfectly conducting scatterer. This augmentation eliminates the spurious resonances without sacrificing the basic simplicity, solution capability, or pure magnetic-field character of the original MFIE. Specifically, the augmented magnetic-field integral equation (AMFIE) takes the form

$$
-\vec{H}_{\text {inc }}(\bar{r})=\frac{1}{2} \hat{n} \times \bar{K}(\bar{r})+\frac{1}{4 \pi} \oint_{S} \bar{K} \times \nabla^{\prime} \psi d S^{\prime} .
$$

where $\bar{K}(\bar{r})$ is the surface current at an observation point $\bar{r}$ on the surface $s$ of the cube, $\bar{H}_{\text {inc }}(\bar{r})$ is the incident magnetic field at that observation point, and $\psi$ is the usual scalar Green's function defined after (10). The original MFIE is reclaimed from the AMFIE merely by crossing the normal $\hat{n}$ out of surface at $\bar{r}$ into both sides of (18). Like the original MFIE, the integral in (18) is evaluated in a surface principal value sense [3].

A desirable feature of the AMFIE (18) is its solvability by the same simple numerical "method of moments" scheme applied to the original MFIE. In particular, when we apply the numerical scheme that Tsai, Dudley, and Wilton [1] used, i.e., the same numerical scheme that we applied to the MFIE to obtain Fig. 6 ,

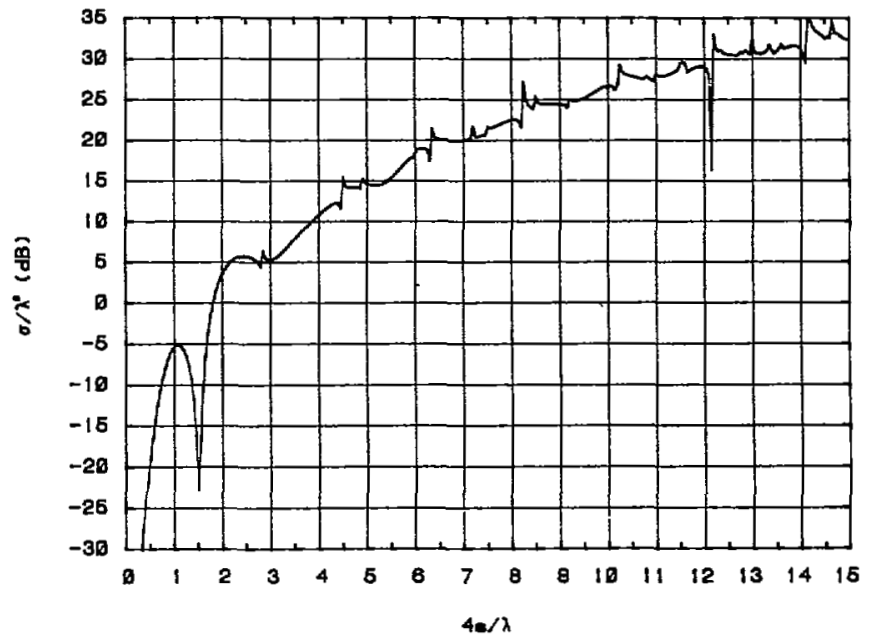

Fig. 6. Broadside RCS computed from MFIE solution.

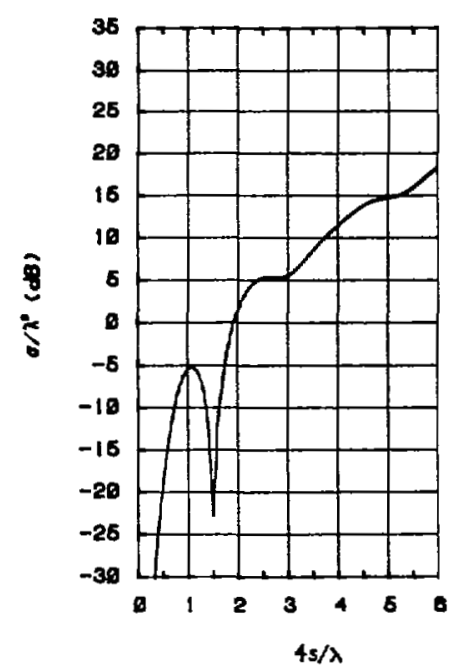

Fig. 7. Broadside RCS computed using AMFIE solution beyond $4 s / \lambda=1.5$.

the RCS curve shown in Fig. 7 results. (Actually, the MFIE is used for $4 s / \lambda \leqslant 1.5$ and the AMFIE for $4 s / \lambda>1.5$ because the MFIE yields slightly greater accuracy at the very low frequencies.) One can see from Fig. 7 that the spurious resonances of Fig. 6 are removed. However, the solution beyond $4 s / \lambda \approx 6$ is not shown because numerical experimentation with patch size showed that acceptable accuracy could be assumed only up to $4 s / \lambda \approx 6$ when limited to a patch size corresponding to 384 patches over the cube. Smaller patch sizes were not possible with the present computer program, because, even using the symmetry of the cube to reduce computer time and storage, the central memory of the available Cyber 750 was not large enough to handle more patches. Also, more than two hours of computer time was required to obtain the curve of Fig. 7. (Such large computer time and storage requirements are the major reasons for the surface integral equations having seen little application to general three-dimensional bodies greater than a couple of wavelengths across.)

\section{THE COMPOSITE SOLUTION}

We have been able to enhance the conventional high frequency solution for the cube in order to reduce the limits of accuracy 
to a fraction of a decibel at frequencies bordering on the dominant resonance. In addition, use of the augmented magnetic field integral equation has extended the accurate range of our integral equation solution to frequencies further above resonance than previous integral equation solutions for broadside backscattering of the conducting cube. In short, the EHFD solution of Fig. 2 and the AMFIE solution of Fig. 7 compare very closely over the intermediate frequency range from $4 s / \lambda \approx 1.6$ to 6 , and thus can be combined to form a composite solution that holds for all frequencies. Fig. 8 plots the composite RCS solution out to $4 s / \lambda$ equal to 15 and compares this theoretical curve with measured RCS data taken at $11 \mathrm{X}$-band frequencies on 18 solid aluminum cubes (pictured in Fig. 11) varying in side length from 5 to 100 $\mathrm{mm}[15]$. The composite curve in Fig. 8 uses the AMFIE solution of Fig. 7 for $4 s / \lambda \leqslant 5.1$ and the EHFD solution of Fig. 2 for $4 s / \lambda>5.1$. The predicted and measured values of RCS compare to within the two-sigma experimental limits of accuracy of about $\pm 1 \mathrm{~dB}$. There is also good agreement with the RCS of the cube computed at $4 s / \lambda=1.27$ by Taflove and Umashankar [16] using both finite-difference-time-domain and EFIE methods.

The composite phase curve of the backscattered far magnetic field referenced to the incident magnetic field on the front face of the cube $(z=0)$ is shown in Fig. 9. At high frequencies the phase approaches $-90^{\circ}$, the far magnetic field phase produced by the physical optics current on the front face of the cube. As the frequency approaches zero the phase of the far magnetic field approaches $-180^{\circ}$ because a quasistatic plane wave incident upon the cube induces an electric dipole moment parallel and in phase with the incident $E$-field, and a magnetic dipole moment parallel and $180^{\circ}$ out of phase with the incident $H$-field. Both these quasi-static dipole moments produce a far magnetic field $180^{\circ}$ out of phase with the incident $H$-field. (This result also follows from the theorem proven in [16] that the copolarized terms in the complex backscattering matrix have zero phase shift.)

Finally, for the sake of comparison with a familiar canonical solution, Fig. 10 shows the RCS versus frequency curve of a perfectly conducting sphere of radius $a$ computed from the exact spherical eigenfunction solution (e.g., see [8, ch. 9]) and plotted to the same scale as Fig. 8. The first resonance of the sphere occurs at nearly the same peximeter and has nearly the same magnitude as that of the cube, but for frequencies beyond the first resonance the strong specular reflection from the front face of the cube raises its RCS well above the RCS of the sphere. It is also of interest to compare the broadside RCS curve of the cube (Fig. 8) with the on-axis RCS versus frequency curves computed for semi-infinite open circular waveguides with and without an internal baffle $\lfloor 17$, figs. 6 and 10].

\section{SUMMARY}

The sparsity of accurate $(\approx \pm 1 \mathrm{~dB})$ broad-band solutions to general, three-dimensional scatterers with edges and corners motivated our looking into the problem of broadside backscattering from the perfectly conducting cube. Existing magneticfield integral equation (MFIE) solutions for the cube encounter spurious resonances that cause serious inaccuracies for cube side lengths greater than about $0.5 \lambda$. Existing GTD solutions for the cube yield an accurate broadside RCS only at considerably higher frequencies where the GTD solution practically coincides with the physical optics integration from the front face of the

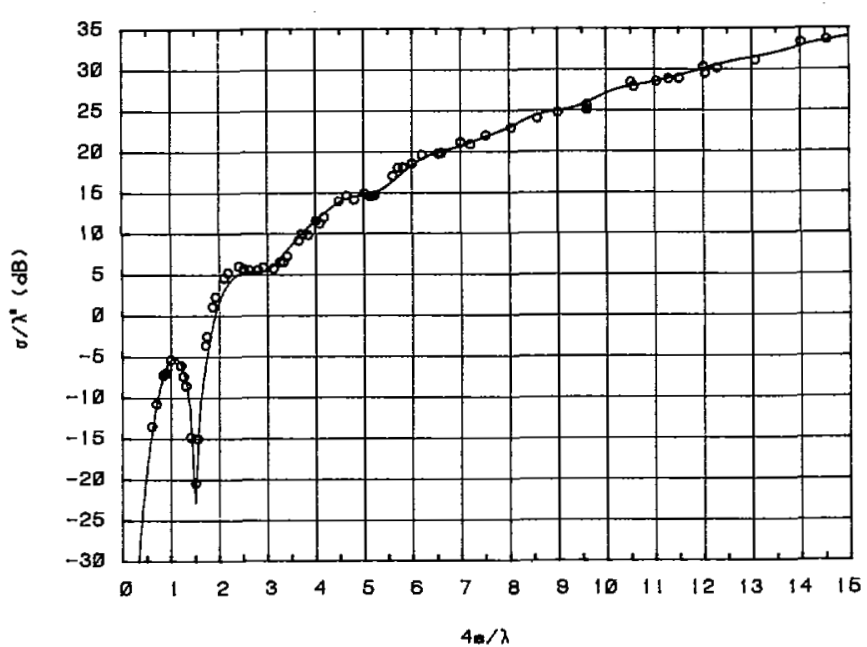

Fig. 8. Broadside RCS computed using composite of AMFIE and EHFD solutions. Circles indicate measured data.

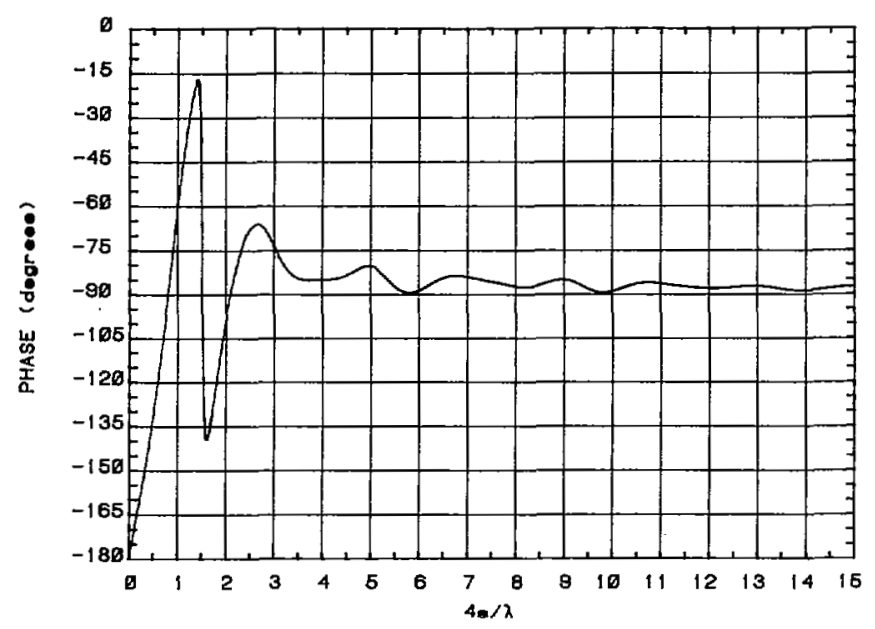

Fig. 9. Phase of the backscattered far magnetic field referenced to the incident magnetic field on the front face of the cube.

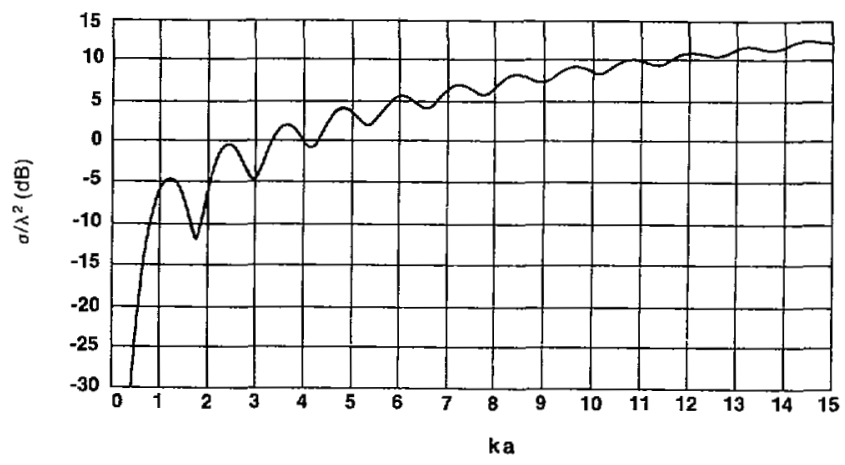

Fig. 10. RCS for perfectly conducting sphere of radius $a$. 


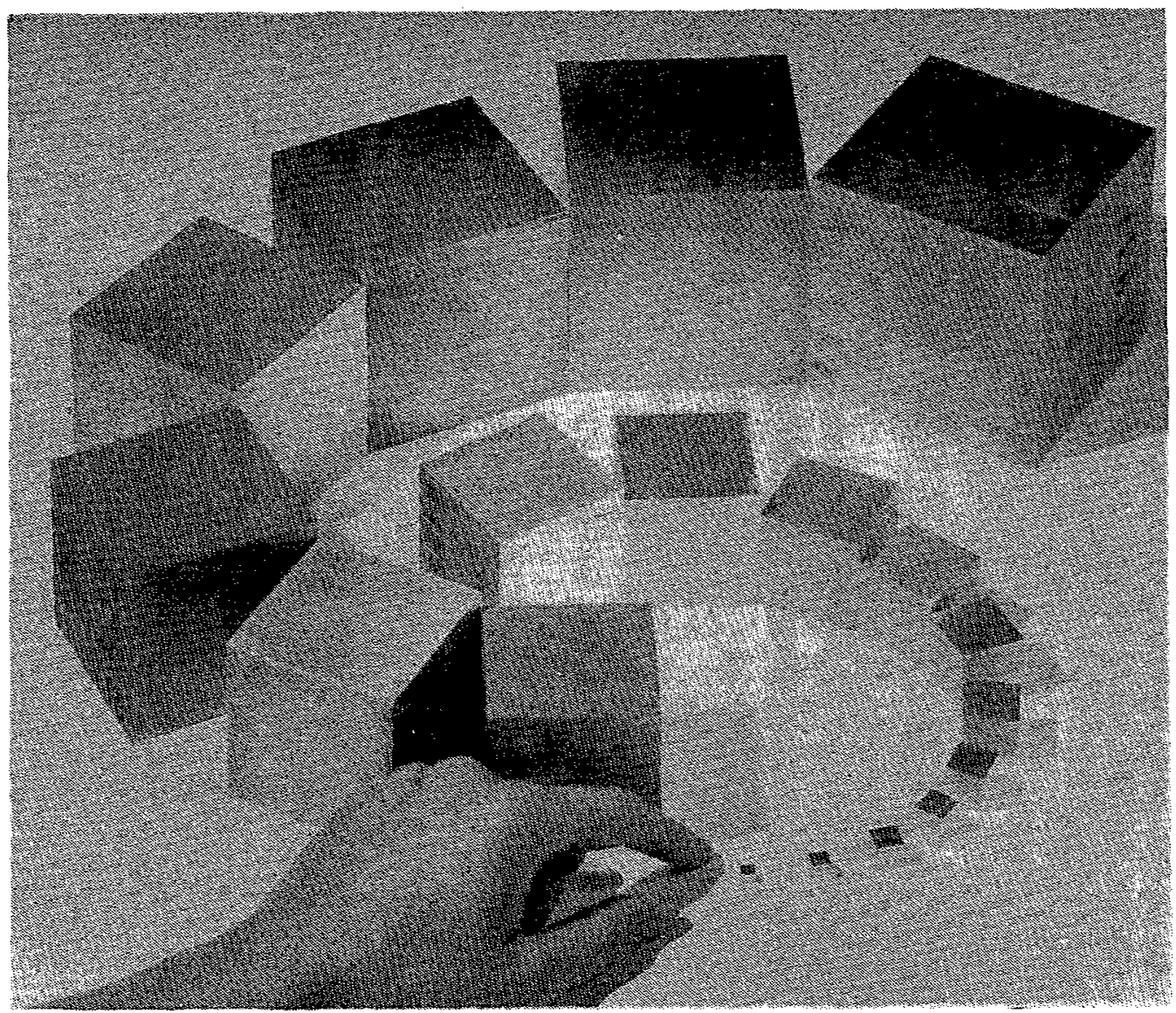

Fig. 11. Eighteen aluminum cubes varying in side lengths from 5 to $11 \mathrm{~mm}$.

cube. Thus, not only is there an intermediate range of frequencies in which no accurate solution exists, but there is also no common range of frequencies that can be used to compare reliably the low frequency integral equation solutions and the high frequency diffraction solutions.

The present approach has been first to develop an enhanced high frequency diffraction solution that determines the RCS of the cube with considerable accuracy down to frequencies in the resonance region; and secondly, to use an augmented magnetic-field integral equation to eliminate the spurious resonances and extend the range of validity of the original MFIE to frequencies well above resonance, i.e. to as high a frequency as available computer time and storage permits. This twofold approach of using the AMFIE and EHFD solutions enabled us to make a mutually confirming comparison of RCS computed by two very different techniques over an intermediate range of frequencies. Moreover, it produced an accurate composite expression for the broadside RCS of the cube at any frequency that compared to within $\pm 1 \mathrm{~dB}$ with measured data taken for the cube side lengths ranging from 0.15 to 4 wavelengths. This accurate composite RCS curve has been used as a benchmark solution to estimate the accuracy and frequency range of validity of general numerical codes that compare scattering [15].

The general development and numerical verification of the augmented integral equations were presented in previous publications[3]. However the technique used to enhance the conventional high frequency diffraction solution is apparently new, although quite simple in essence and straightforward in applica- tion. Instead of using the conventional GTD approximation for the grazing fields impinging upon the trailing edges of the cube, we computed the scattered part of the impinging fields by integrating over the physical optics and fringe currents of the front face and edges of the cube. In other words, the currents induced on the leading face and edges of the cube were used to better estimate the fields impinging upon the trailing edges. This refinement increased considerably the accuracy of the solution for cube side lengths less than one wavelength, and produced a high frequency diffraction curve that agreed closely with the integral equation solution at intermediate frequencies. Unlike the conventional high frequency solutions, the EHFD solution clearly revealed the dominant resonance of the cube that peaks at a cube side length of approximately 0.25 wavelengths.

Finally, this enhancement technique can also be applied to perfectly conducting scatterers other than the cube, provided a definite separation exists between leading and trailing ends of the scatterer along the direction of propagation of the incident field. Whether the technique is feasible for more general geometries and aspect angles remains to be determined.

\section{REFERENCES}

[1] L L. Tsai, D. G. Dudley, and D. R. Wilton, "Electromagnetic scattering by a three-dimensional conducting box," J. Appl. Phys., vol. 45, no. 10, pp. $4393-4400$, Oct. 1974.

[2] C. E. Ryan, Jr., "Diffraction analysis of scattering by a cube with application to the time response waveforms," The Ohio State Univ. ElectroSci. Lab. Rep. 2415-3, Mar. 1970.

[3] A. D. Yaghjian, "Augmented electric- and magnetic-field integral 
equations," Radio Sci., vol. 16, no. 6, pp. 987-1001, Nov.-Dec., 1981 (See also RADC TR-81-45 under the same title, Mar. 1981.)

[4] C. E. Ryan, Jr., "Time-response waveforms for a cube from measured data and diffraction analysis," Radio Sci., vol. 6, no. 8-9, pp. 801-804, Aug.-Sept., 1971.

[5] P. Ia. Ufimtsev, "Approximate computation of the diffraction of plane electromagnetic waves at certain metal bodies," Sov. Phys.-Tech. Phys., vol. 27, no. 8, pp. 1708-1718, Aug. 1957.

[6] C. E. Ryan, Jr. and L. Peters, Jr., "Evaluation of edge-diffracted fields including equivalent currents for the caustic regions," IEEE Trans. Antennas Propagat., vol. AP-17, no. 3, pp. 292-299, May, 1969.

[7] E. F. Knott and T. B. A. Senior, "Comparison of three high-frequency diffraction techniques," Proc. IEEE, vol. 62, no. 11, pp. 1468-1474, Nov., 1974.

[8] J. J. Bowman, T. B. A. Senior, and P. L. E. Uslenghi, Electromagnetic and Acoustic Scattering by Simple Shapes. Amsterdam: NorthHolland, 1969, ch. 6 .

[9] F. A. Sikta, W. D. Burnside, T. T. Chu, and L. Peters, Jr., "First-order equivalent current and corner diffraction scattering from flat plate structures," IEEE Trans. Antennas Propagat., vol. AP-31, no. 4, pp. 584-589, July, 1983.

[10] R. E. Collin and F. J. Zucker, Antenna Theory, part 1. New York: McGraw-Hill, 1969, eq. (2.23a).

[11] R. G. Kouyoumjian, "The geometrical theory of diffraction and its application," in Numerical and Asymptotic Techniques in Electromagnetics (Topics in Applied Physics, vol. 3), R. Mittra, Ed. New York: Springer-Verlag, 1975.

[12] J. J. H. Wang and C. J. Drane, "Numerical analysis of arbitrarily shaped bodies modeled by surface patches," IEEE Trans. on Microwave Theory Tech., vol. MTT-30, no. 8, pp. 1167-1173, Aug. 1982.

[13] F. H. Murray, "Conductors in an electromagnetic field, $\left(\mathrm{E}^{\circ} \mathrm{e}^{\mathrm{pt}}, \mathrm{H}^{\mathrm{o}} \mathrm{e}^{\mathrm{pt}}\right)$," Amer. J. Math., vol. 53, no. 2, pp. 275-288, 1931.
[14] A. W. Maue, "On the formulation of a general scattering problem by means of an integral equation," Z. Phys., vol. 126, no. 7, pp. 601-618, Aug. 1949.

[15] R. V. McGahan, "Scattering experiments at the Ipswich Electromagnetic Measurements Facility: Backscatter from metal cubes," RADC TR-85-24, Feb. 1985.

[16] W. B. Goggins, Jr., P. Blacksmith, and C. J.Sletten, "Phase signature radars," IEEE Trans. Antennas Propagat., vol. AP-22, no. 6, pp. 774-780, Nov. 1974.

[17] T. W. Johnson and D. L. Moffatt, "Electromagnetic scattering by open circular waveguides," Radio Sci., vol. 17, no. 6, pp. 1547-1556, Nov.Dec. 1982.

Arthur D. Yaghjian (S'68-M'69-SM'84), for a photograph and biography please see page 5 of the January 1984 issue of this TRANSACTIONS.

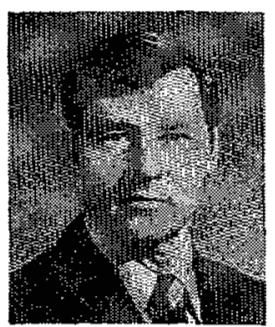

Robert V. MeGahan (S'74-M'75) received the B.S. degree with high honors from the Lowell Technological Institute, Lowell, MA in 1972, and the S.M. degree from the Massachusetts Institute of Technology, Cambridge, in 1975, both in electrical engineering.

Since 1974, he has worked for the Air Force Cambridge Research Laboratories and for the Rome Air Development Center at Hanscom Air Force Base on phased arrays, limited scan antennas, scattering, and radar cross section mesurements. and Propagation Society and Eta Kappu Nu. 